\title{
NOTES
}

\section{Improvement in the Compositional Analysis of Block Copolymers of Ordinary and Deuterated Styrenes by High-Resolution Pyrolysis Gas Chromatography}

\author{
Hajime Ohtani, Shin Tsuge, * Yuhshuh Matsushita, \\ and Mitsuru NAGASAWA \\ Department of Synthetic Chemistry, Faculty of Engineering, \\ Nagoya University, Nagoya 464, Japan
}

(Received March 19, 1984)

\author{
KEY WORDS Pyrolysis Gas Chromatography / Fused Silica Capillary \\ Column / Polystyrene / Poly(styrene- $b$-styrene- $d_{8}$ )
}

Partially deuterium labelled polystyrenes are particular samples for observing the molecular motion of a part of the polymer chain by small angle neutron scattering. In these polymers special attention should be paid to sample characterization, especially the accurate determination of deuterium content. Recently, we proposed a method for determining deuterated block content in the block copolymers of styrene and styrene- $d_{8}$ using pyrolysis gas chromatography (PGC). ${ }^{1}$ Since this method requires minimal sample size $(0.03$ to $0.1 \mathrm{mg}$ ) and simple operating procedure, it is suited for the analysis of deuterated polymers prepared in small quantity. However, even if a glass capillary column is used the complete peak separation of deuterated and the ordinary hydrogenated products can be attained only for trimers. Therefore, trimers with relatively small yield (about 10\%) had to use for determining deuterated block content. Moreover, the difference in the relative yields of the trimers from styrene- $d_{8}$ sequences (trimer- $d$ ) and from ordinary styrene sequences (trimer- $h$ ) must be corrected empirically by measuring the pyrogram of associated homopolymers of comparable molecular weight. Accordingly, the precision of such de-

\footnotetext{
* To whom correspondence should be addressed.
}

termination is not necessarily sufficient.

In this work, almost a complete separation was achieved even for the main characteristic peaks such as those of monomers, dimers, and trimers on pyrograms of the block copolymers of styrene and styrene- $d_{8}$ by PGC used in junction with a new high-resolution fused silica capillary. By this method, separated characteristic products such as monomers, dimers and trimers were totally evaluated for their cotnent in block copolymers. Consequently, the precision of analysis had been greatly improved.

\section{EXPERIMENTAL}

\section{Materials}

Two kinds of block copolymers of poly(styrene- $b$-styrene- $\left.d_{8}\right)$, SD-1 $\left(M=2.78 \times 10^{5}\right)$ and SD-2 $\left(M=1.96 \times 10^{5}\right)$, were synthesized by the sequential monomer addition technique of anionic polymerization. ${ }^{2}$ Two homopolymers, poly(styrene- $d_{8}$ ) (PSt- $d$ ) with $M_{n}=$ $2.7 \times 10^{4}$ and polystyrene (PSt- $h$ ) with $M_{n}=$ $1.4 \times 10^{5}$ were used for the calibration.

\section{Conditions for PGC}

A vertical microfurnace-type pyrolyzer 


\section{H. Ohtani et al.}

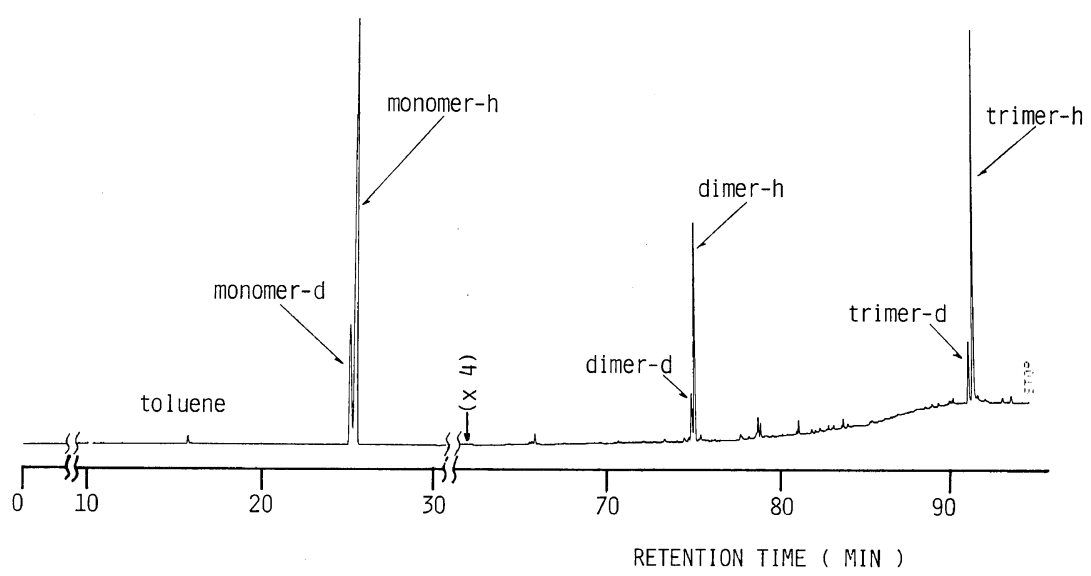

Figure 1. Pyrogram of a poly(styrene- $b$-styrene- $\left.d_{8}\right)(\mathrm{SD}-2)$ at $500^{\circ} \mathrm{C}$.

(Yanagimoto GP-1018) was directly attached to a gas chromatograph (Shimadzu 7AG) with a fused silica open tubular capillary column (0.32-mm o.d. $\times 0.2-\mathrm{mm} \quad$ i.d. $\times 30-\mathrm{m}$ long) coated by immobilized OV-1 (polydimethylsiloxane) through chemical crosslinking supplied by Hewlett Packard. The flow rate of the nitrogen carrier gas was reduced from $50 \mathrm{ml}$

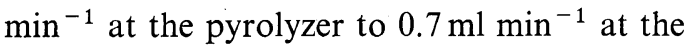
capillary column by a splitter $(70: 1)$. The column temperature was held initially at $40^{\circ} \mathrm{C}$ for $2 \mathrm{~min}$, and raised at a rate of $1.5^{\circ} \mathrm{C} \mathrm{min}{ }^{-1}$ to $80^{\circ} \mathrm{C}$, then $2.5^{\circ} \mathrm{C} \mathrm{min}^{-1}$ to $200^{\circ} \mathrm{C}$, finally

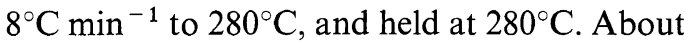
a $30 \mu \mathrm{g}$ sample was pyrolyzed at $500^{\circ} \mathrm{C}$ under the flow of the carrier gas. Peak identification was made primarily through use of a mass spectrometer, model D-300 (JEOLCO) coupled directly to the gas chromatograph.

\section{RESULTS AND DISCUSSION}

Figure 1 shows a typical high-resolution pyrogram for poly(styrene- $b$-styrene- $d_{8}$ ) obtained by the fused silica capillary column. The main peaks of the pyrogram, i.e., those of the monomer, dimer, and trimer peaks, are doublets each consisting of a deuterated $(d)$ and hydrogenated $(h)$ pair. Hybrid fragments such as $d h$, $d d h$, dhd, hhd, and $h d h$, which are
Table I. The content of $d$-blocks in the copolymers

\begin{tabular}{|c|c|c|}
\hline \multirow{2}{*}{ Sample } & \multicolumn{2}{|c|}{ Content of $d$-block $/ \mathrm{mol} \% \mathrm{a}$} \\
\hline & By this method ${ }^{\mathrm{b}}$ & By previous method ${ }^{\mathrm{c}}$ \\
\hline SD-1 & $19.1( \pm 0.1)$ & $19.6( \pm 0.5)$ \\
\hline $\mathrm{SD}-2$ & $20.7( \pm 0.1)$ & $21.1( \pm 0.5)$ \\
\hline
\end{tabular}

a Results based on at least four determinations.

b Calculated from eq 1.

c Obtained using relative trimer yields.

expected to be present in trace amounts even in the block copolymers, could not be found under the experimental conditions. Therefore, the following discussion assumes that the relative yields of hybrid fragments, if any, are negligibly small for block copolymer sample with $M_{n}>10^{5}$. Other small peaks such as toluene, etc. were also observed on the pyrogram. The relative abundance of the minor components, however, indicated slightly different values for the associated homopolymers; $1.9 \%$ and $3.5 \%$ for PSt- $d$ and PSt- $h$, respectively, where the total peak intensity of the monomer, dimer and trimer plus the minor components in each case is regarded as $100 \%$. These data suggest the total peak intensities of the monomer, dimer, and trimer to be $98.1 \%$ and $96.5 \%$ for PSt- $d$ and PSt- $h$, respectively. 
Considering this, the deuterated block content in the copolymer can be calculated as follows:

$d$-content $\left(\mathrm{mol}^{\mathrm{o}} \mathrm{o}\right)=\frac{\frac{I_{d-(m+d+1)}}{0.981}}{\frac{I_{d-(m+d+t)}}{0.981}+\frac{I_{n-(m+d+t)}}{0.965}} \times 100$

where $I_{d-(m+d+t)}$ and $I_{h-(m+d+h)}$ are the total peak intensities of the $d$-monomer, $d$-dimer, and $d$-trimer, and those of the $h$-monomer, $h$ dimer, and $h$-trimer from the copolymers.

The results obtained by this method are given in Table I along with those by the previous method ${ }^{1}$ using only the trimer yields. The data in parentheses show the range of fluctuation for four measurements. It can be seen that the precision of analysis is greatly improved as a result of using the major characteristic peaks of the monomers, dimers, and trimers. In practice, both correction factors for PSt- $d$ (0.981) and PSt- $h(0.965)$ are close to unity. Thus, even if the peak intensities are not corrected by these factors, the resulting relative deviation is restricted to within $2 \%$.

\section{REFERENCES}

1. H. Ohtani, S. Tsuge, Y. Matsushita, and M. Nagasawa, Polym. J., 14, 495 (1982).

2. Y. Matsushita, H. Furuhashi, H. Choshi, I. Noda, M. Nagasawa, T. Fujimoto, and C. C. Han, Polym. J., 14, 489 (1982). 